\title{
CAUDALES Y VARIABILIDAD CLIMÁTICA EN UNA CUENCA DE LATITUDES MEDIAS EN SUDAMÉRICA: RÍO ACONCAGUA, CHILE CENTRAL $\left(33^{\circ} \mathrm{S}\right)$
}

\author{
Carolina Martínez ${ }^{1}$, Alfonso Fernández'1, Patricio Rubio² \\ ${ }^{1}$ Departamento de Geografía, Universidad de Concepción. Chile \\ 2Departamento de Geografía Física y Análisis Geográfico Regional. Universidad de Barcelona
}

\section{RESUMEN}

Se analizan caudales de estaciones fluviométricas en la cuenca del río Aconcagua (Chile Central) y su asociación con la variabilidad climática inducida por eventos ENOS (período 1950-2000). Se determinaron correlaciones lineales entre tendencias anuales y estacionales de los caudales e índices ENSO, a través del índice de la temperatura superficial del mar (TSM) de la región Niño 3.4. El 50\% de las estaciones fluviométricas se encontraron con déficit hídrico de hasta $11,3 \%$ y el 50\% restante tienden al superávit. Se encontró asociación entre los caudales máximos mensuales y eventos El Niño, caudales mínimos mensuales con eventos La Niña y correlaciones positivas entre el índice TSM y los caudales medios mensuales para el mismo período estacional.

Palabras clave: cuenca hidrográfica, ENOS, caudal, variabilidad climática, río Aconcagua, Chile

\section{ABSTRACT}

Stream flow of the Río Aconcagua basin (Central Chile) and its relationship with climatic variability of the ENSO was analyzed for the period 1950-2000. Linear trends of winter and summer seasons were performed. Yearly and seasonal trends were correlated with the Sea Surface Temperature (SST) index of the ENSO 3.4 zone. While $50 \%$ of the fluviometric stations had a hydrological deficit which reached $11.3 \%$, the other $50 \%$ recorded a surplus. We found associations between the maximum mean monthly flow and El Niño events,

Fecha de recepción: julio 2010.

Fecha de aceptación: febrero 2012. 
minimum monthly mean flow and La Niña events, and a positive correlation between the SST index and the mean monthly flow for the period.

Key words: river basin, ENSO, water resources, stream flow, climatic variability, Aconcagua river, Chile.

\section{INTRODUCCIÓN}

La cuenca hidrográfica exorreica del río Aconcagua es una de las más importantes de Chile semiárido, no solo por la superficie drenada $\left(7.163 \mathrm{~km}^{2}\right)$ que equivale al $45 \%$ de la superficie de la Región de Valparaíso, sino por la relevancia económica que representa a nivel regional, porque gran parte del Producto Interno Bruto recae en las actividades agropecuarias e industriales que se realizan en torno al curso medio e inferior del río. Por lo tanto, la disponibilidad de agua, es un aspecto esencial para planificar el desarrollo económico regional en términos de sustentabilidad, en especial si se considera que la demanda para riego, industria, minería y uso doméstico supera los 500 millones de $\mathrm{m}^{3}$ de agua al año, principalmente en la zona media e inferior del valle donde habita casi el 30\% de la población regional. Los recursos disponibles para satisfacer esta demanda de agua provienen de cauces superficiales y aguas subterráneas, con una infraestructura de riego de 1.230 canales y 53 embalses, estos en su mayoría localizados en el curso inferior de la cuenca. En el contexto de la gestión integrada de cuencas hidrográficas, el conocimiento sobre las características del régimen hidrológico y la variabilidad de los caudales en relación con fenómenos interdecadales es un tema central para proyectar las acciones futuras en relación con el uso del agua.

Con el propósito de contribuir a este conocimiento, se analiza aquí el comportamiento de los caudales en la cuenca del río Aconcagua para el período 1961-2000 y su relación con los factores que influyen en la variabilidad climática de Chile Central, dado que algunos estudios en el país han establecido una disminución progresiva de la capa de nieve que alimenta a las cuencas andinas de régimen mixto como la del río Aconcagua, lo cual es concordante con otros estudios sobre tendencias mundiales. Así también, se ha establecido una fuerte asociación entre precipitaciones y variabilidad climática para estaciones meteorológicas de Chile Central (Aceituno y Vidal, 1990; Escobar y Aceituno, 1998; Caviedes y Waylen, 1998), así como entre caudales y fases ENOS (Waylen y Caviedes, 1990).

El objetivo de este trabajo es (I) analizar el comportamiento histórico de los caudales en la cuenca del río Aconcagua de Chile central, para el período 1961-2000 y, (II) analizar la relación entre caudal y fenómeno ENSO en la cuenca del río Aconcagua. Se propone aquí que los factores que influyen en la variabilidad climática pueden ser expresados a través de relaciones de dependencia entre caudales y temperatura superficial del mar (TSM), las cuales pueden consideradas variables síntesis que facilitan la interpretación sistémica sobre la variabilidad climática y como vía para mejorar la toma de decisiones en relación con la planificación y gestión de los recursos hídricos en cuencas hidrográficas. 


\section{II. ÁREA DE ESTUDIO}

El río Aconcagua nace en la cordillera de Los Andes, a través de la conjunción de los ríos Juncal y Blanco a una altura entre 5.400 y 7.000 msnm conformando una amplia red de drenaje de unos $7.000 \mathrm{~km}^{2}$ y describiendo un recorrido de $190 \mathrm{~km}$ hasta desembocar en la bahía de Concón, en la Región de Valparaíso (33으) (Fig. 1). A $1.420 \mathrm{msnm}$ el caudal registra un promedio anual de $20,5 \mathrm{~m}^{3} / \mathrm{s}$ y a unos $600 \mathrm{msnm}$ (sector Las Vizcachas) registra unos 33 $\mathrm{m}^{3} / \mathrm{s}$ (DGA, 2004).

De acuerdo a las características físicas de la cuenca, los regimenes de escurrimiento, gasto de los ríos y caudales comprometidos, Niemeyer y Cereceda (1984) clasifican al sistema hidrológico del río Aconcagua dentro de la Segunda Zona Hidrográfica o «Ríos en torrente, de régimen mixto en la zona semiárida de Chile», caracterizada por fuertes pendientes y considerables diferencias altimétricas que influencian el comportamiento en torrente. Esta zona se caracteriza por presentar un relieve montañoso irregular que forma un solo bloque al asociar a la Cordillera de Los Andes y de la Costa, cortado de oriente a poniente por una serie de valles tectónicos que controlan en gran parte los cursos fluviales. Es la única zona del país que carece de volcanismo y presenta además las mayores altitudes andinas, lo cual condiciona que el escurrimiento de los ríos sea en torrente, alcanzándose pendientes superiores al 2,5\% entre el curso superior y el mar. La gran variabilidad anual de los gastos es otra característica importante de esta zona (Niemeyer y Cereceda, 1984).

Desde el punto de vista geológico, la cordillera de La Costa desde la cuesta de Barriga al valle de La Ligua $\left(32^{\circ} 20^{\prime}-32^{\circ} 35^{\prime}\right.$ y $70^{\circ} 40^{\prime}-71^{\circ} 30^{\prime} \mathrm{W}$ ) se compone principalmente de estratos del Mesozoico cuyas edades fluctúan entre el Triásico superior al Cretácico superior y que presenta un buzamiento general hacia el Este (Thomas, 1958). El autor indica además que cerca de la costa aparecen afloramientos aislados fuertemente plegados como neises, micacitas y filitas de edad probable paleozoica o prepaleozoica. Todas las formaciones están atravesadas, a excepción de los sedimentos terciarios, por intrusiones de granodiorita y diorita que presentan localmente facies más ácidas o más básicas. Desde el punto de vista biogeográfico, la cuenca del río Aconcagua se caracteriza por asociaciones vegetales típicas de clima mediterráneo, presentando las siguientes comunidades vegetales: Matorral esclerófilo andino (zona alta del río); Matorral Espinoso de las serranías y Bosque esclerófilo costero (valle y desembocadura del río respectivamente) (Gajardo, 1994).

\section{MATERIALES Y MÉTODOS}

\section{Definición del período de análisis y depuración de la base de datos}

Para analizar el comportamiento de los caudales en la cuenca del río Aconcagua, se utilizaron datos de caudales medios mensuales y anuales registrados en las 13 estaciones fluviométricas que se localizan en el área de estudio. Éstas pertenecen a la base de datos de la Dirección General de Aguas del Ministerio de Obras Públicas de Chile (DGA-MOP) (Fig. 1). De estas, 11 fueron utilizadas para el análisis estadístico-descriptivo con series de tiempo entre 1950 y 2000 (Cuadro 1). Las estaciones 1 y 8 no fueron consideradas. Para el análisis de tendencias fue necesario estandarizar los datos con el objeto de establecer un período 
de análisis homogéneo, dado que las estaciones localizadas en la sección media y alta de la cuenca cuentan con series de tiempo mayores y períodos coincidentes. De este modo, se redujo a estaciones que presentaron registros coincidentes, acotando el período a 1961-2000. Adicionalmente, la base de datos registró una serie de datos rellenados, que fluctúan entre $26 \%$ (Río Blanco en Río Blanco) a 1.9 \% (Río Aconcagua en Chacabuquito) para todo el período. Se decidió eliminar aquellos registros con más de un año sin información dentro del período de análisis común. Esto implicó que las estaciones 4, 5, 7, 9, 10, 11, 12 y 13 (Cuadro 1) fueran seleccionadas.

Debido a que la cuenca del río Aconcagua está altamente antropizada, fue necesario identificar adicionalmente las estaciones que podrían estar asociadas a modificaciones humanas del ciclo anual, distorsionando su respuesta frente a fenómenos de influencia interanual. Para realizar este filtro, se analizó la distribución anual de los caudales sobre la base de histogramas de frecuencias relativas y su posición con respecto a los embalses, tomando la base de datos de la DGA como fuente.

Como se observa en la Fig. 2, todas las estaciones guardan un comportamiento similar, mostrando los máximos caudales después de octubre, mientras que los mínimos entre abril y septiembre. La única excepción correspondió a la estación $\mathrm{N}^{0} 4$, que mostró sus máximos entre mayo y noviembre. Su cercanía a embalses puede indicar su diferente comportamiento. De esta forma, el análisis consideró sólo 7 estaciones fluviométricas.

\section{Caudales mensuales}

Los caudales medios mensuales fueron analizados de dos formas. En primer lugar, se realizó un análisis descriptivo a partir de la distribución de caudales anuales, sus máximos en el período de análisis y sus tiempos de recurrencia. Para esto último, se aplicó el test de Kolmogorov-Smirnov para determinar el ajuste de las distribuciones reales de caudales máximos con las distribuciones de probabilidad Gaussiana, Lognormal, Gumbel y Pearson III, consideradas las más útiles para estos casos (Aparicio, 1992). En segundo lugar, se analizaron las tendencias lineales de los caudales medios anuales para las estaciones de verano (DEF) e invierno (JJA). La significancia estadística de las tendencias fue determinada aplicando el test de Student de dos colas.

\section{Correlación con el ENSO}

Finalmente, se determinaron correlaciones lineales entre las tendencias anuales y estacionales de los caudales y los índices que describen el ENSO. Para esto, se utilizó el índice de la temperatura superficial del mar (TSM) de la NOAA (http://www.cpc.ncep.noaa.gov) para la región Niño 3.4 , localizada entre los $5^{\circ} \mathrm{N}-5^{\circ} \mathrm{S}$ y los $120^{\circ}-170^{\circ} \mathrm{O}$ (Trenberth, 1997). Las correlaciones se realizaron en los pares TSM-Caudales durante la misma estación, TSM verano-Caudal invierno consecutivo y TSM invierno-Caudal verano del año siguiente. Todas estas comparaciones están asociadas a fechas de año civil. 
Cuadro 1

ESTACIONES FLUVIOMÉTRICAS DE LA CUENCA DEL RIOO ACONCAGUA

\begin{tabular}{|c|c|c|c|c|c|c|c|}
\hline Id & Estación & Localización & $\begin{array}{c}\text { Altura } \\
(\mathrm{msnm})\end{array}$ & Serie & $\begin{array}{l}\text { Código } \\
\text { estación }\end{array}$ & $\begin{array}{l}\text { Área } \\
\text { km² }^{2}\end{array}$ & $\begin{array}{c}\text { Datos } \\
\text { rellenados } \\
(\%)\end{array}$ \\
\hline 1 & $\begin{array}{l}\text { Canal las Vegas } \\
\text { en Bocatoma }\end{array}$ & $\begin{array}{l}32^{\circ} 49^{\prime} \mathrm{S}- \\
70^{\circ} 56^{\prime} \mathrm{S}\end{array}$ & 550 & $1970-2000$ & 05420002-1 & 90 & 0 \\
\hline 2 & $\begin{array}{l}\text { Estero Catemu } \\
\text { en Puente Santa } \\
\text { Rosa }\end{array}$ & $\begin{array}{l}32^{\circ} 49^{\prime} \mathrm{S}- \\
70^{\circ} 00^{\prime} \mathrm{S}\end{array}$ & 510 & $1970-2000$ & 05421001-9 & 100 & 0 \\
\hline 3 & $\begin{array}{l}\text { Río Aconcagua en } \\
\text { Romeral }\end{array}$ & $\begin{array}{l}32^{\circ} 50^{\prime} \mathrm{S}- \\
71^{\circ} 01^{\prime} \mathrm{S}\end{array}$ & 310 & $1961-1990$ & 05423003-6 & 5476 & 0 \\
\hline 4 & $\begin{array}{l}\text { Estero Pocuro en } \\
\text { el Sifón }\end{array}$ & & & & & & 10.5 \\
\hline 5 & $\begin{array}{l}\text { Río Aconcagua en } \\
\text { Chacabuquito }\end{array}$ & $\begin{array}{l}32^{\circ} 50^{\prime} \mathrm{S}- \\
70^{\circ} 34^{\prime} \mathrm{S}\end{array}$ & 1030 & $1950-2000$ & 05410002-7 & 2400 & 1.9 \\
\hline 6 & $\begin{array}{l}\text { Río Aconcagua en } \\
\text { Puente Colmo }\end{array}$ & $\begin{array}{l}32^{\circ} 55^{\prime} \mathrm{S}- \\
71^{\circ} 25^{\prime} \mathrm{S}\end{array}$ & 20 & $1997-2002$ & $05426003-2$ & 0 & 0 \\
\hline 7 & $\begin{array}{l}\text { Río Aconcagua en } \\
\text { Río Blanco }\end{array}$ & $\begin{array}{l}32^{\circ} 55^{\prime} \mathrm{S}- \\
70^{\circ} 19^{\prime} \mathrm{S}\end{array}$ & 1420 & $1960-2000$ & 05402001-5 & 382 & 23 \\
\hline 8 & $\begin{array}{l}\text { Estero las } \\
\text { Vegas en } \\
\text { Desembocadura }\end{array}$ & $\begin{array}{l}32^{\circ} 56^{\prime} \mathrm{S}- \\
71^{\circ} 01^{\prime} \mathrm{S}\end{array}$ & 315 & $1970-2000$ & 05422000-1 & 154 & 0 \\
\hline 9 & $\begin{array}{l}\text { Río Aconcagua en } \\
\text { San Felipe }\end{array}$ & $\begin{array}{l}32^{\circ} 45^{\prime} \mathrm{S}- \\
70^{\circ} 45^{\prime} \mathrm{S}\end{array}$ & 630 & $1961-2000$ & 05410005-1 & 3005 & 25 \\
\hline 10 & $\begin{array}{l}\text { Río Blanco en Río } \\
\text { Blanco }\end{array}$ & & & & & & 26 \\
\hline 11 & $\begin{array}{l}\text { Río Colorado en } \\
\text { Colorado }\end{array}$ & $\begin{array}{l}32^{\circ} 52^{\prime} \mathrm{S}- \\
70^{\circ} 25^{\prime} \mathrm{S}\end{array}$ & 1062 & $1960-2000$ & 05406001-7 & 743 & 21 \\
\hline 12 & $\begin{array}{l}\text { Río Juncal en } \\
\text { Juncal }\end{array}$ & $\begin{array}{l}32^{\circ} 53^{\prime} \mathrm{S}- \\
70^{\circ} 09^{\prime} \mathrm{S}\end{array}$ & 1800 & $1960-2000$ & 05401003-6 & 233 & 11.4 \\
\hline 13 & $\begin{array}{l}\text { Río Putaendo en } \\
\text { Resguardo Los } \\
\text { Patos }\end{array}$ & $\begin{array}{l}32^{\circ} 31^{\prime} \mathrm{S}- \\
70^{\circ} 36^{\prime} \mathrm{S}\end{array}$ & 1218 & $1950-2000$ & 05414001-0 & 927 & 8 \\
\hline
\end{tabular}


Figura 1

ÁREADE ESTUDIO Y LOCALIZACIÓN DE LAS ESTACIONES FLUVIOMÉTRICAS
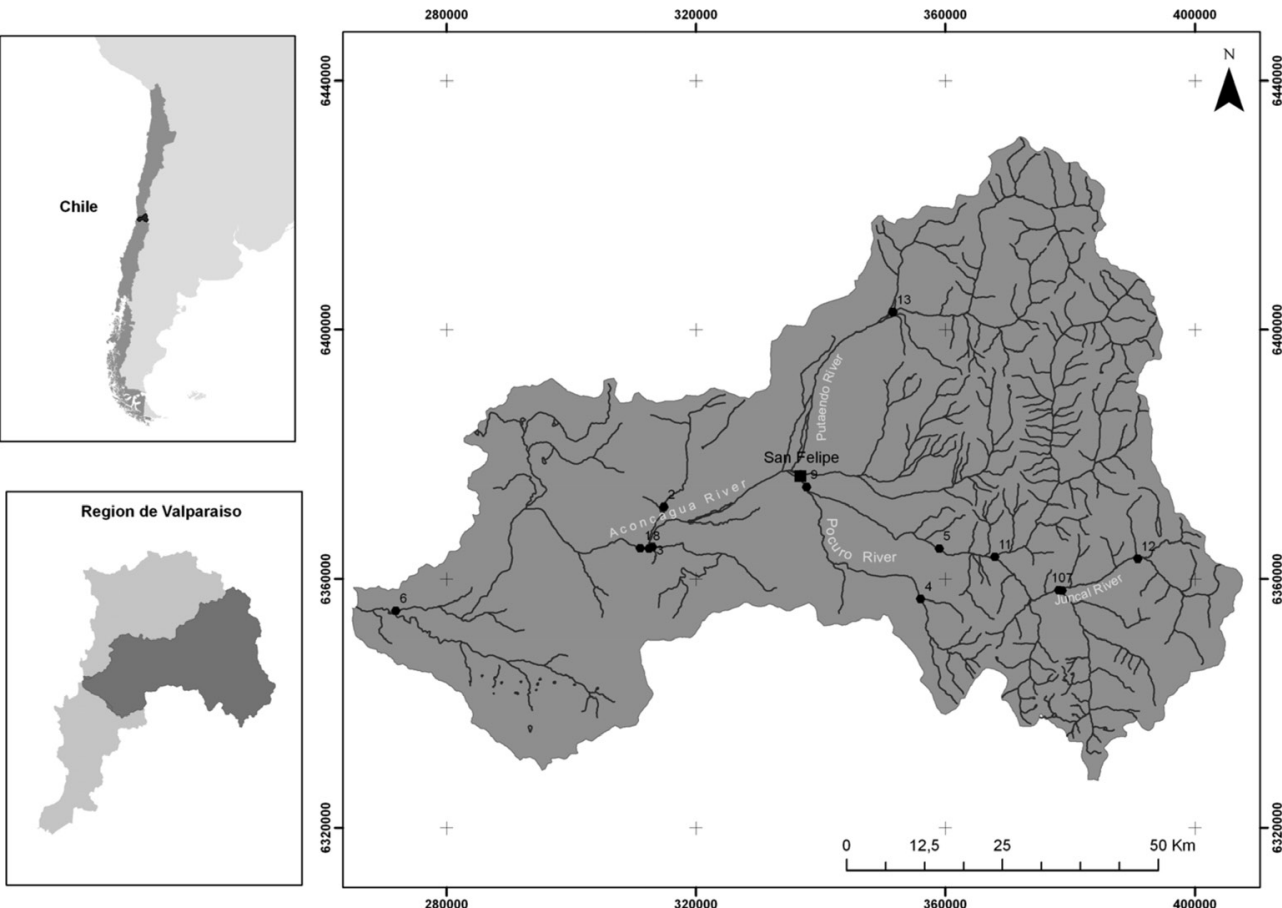

\section{RESULTADOS}

\section{Caudales}

En el curso superior de la cuenca del río Aconcagua, los aportes de precipitación se realizan en forma de nieve con un período de deshielos coincidente con la estación estival, lo cual ocurre entre los meses de septiembre a enero con caudales máximos entre diciembre y enero. En el caso del río Juncal, las variaciones de caudal son importantes durante las crecidas por deshielos, no así durante el resto del año hidrológico donde mensualmente son del orden de 5 $\mathrm{m}^{3} / \mathrm{s}$ y de $70 \mathrm{~m}^{3} / \mathrm{s}$ anuales (Cuadros 2 y 3 ). Los caudales medios mensuales máximos históricos (Cuadro 4) se registraron en el mes de enero de los años $1979\left(30 \mathrm{~m}^{3} / \mathrm{s}\right), 1983\left(25 \mathrm{~m}^{3} / \mathrm{s}\right)$, $1965\left(20 \mathrm{~m}^{3} / \mathrm{s}\right)$ y $1998\left(17 \mathrm{~m}^{3} / \mathrm{s}\right)$.

El afluente del río Blanco en el curso superior del Aconcagua, presentó igual régimen pero con variaciones importantes respecto a los caudales mensuales máximos, con un promedio anual de $224 \mathrm{~m}^{3} / \mathrm{s}$. Según el comportamiento en diferentes décadas (Cuadros 5 y 6), los valores máximos se registraron durante los meses de enero de los años 1973 y 1983 (90 $\left.\mathrm{m}^{3} / \mathrm{s}\right) ; 1964\left(73 \mathrm{~m}^{3} / \mathrm{s}\right)$ y $1992\left(60 \mathrm{~m}^{3} / \mathrm{s}\right)$. En la estación Chacabuquito se observó igual tendencia respecto al régimen hidrológico, la cual es más acentuada en los decenios 1981-1990 y 
Cuadro 2

CAUDALES MEDIOS ANUALES, MÍNIMOS Y MÁXIMOS HISTÓRICOS, CUENCA RÍO ACONCAGUA

\begin{tabular}{|c|c|c|c|c|c|c|}
\hline \multirow{2}{*}{ Estación } & \multirow{2}{*}{ Serie } & \multirow{2}{*}{$\begin{array}{c}\text { Promedio } \\
\left(\mathbf{m}^{3} / \mathbf{s}\right)\end{array}$} & \multicolumn{4}{|c|}{ Caudal anual $\left(\mathrm{m}^{3} / \mathrm{s}\right)$} \\
\hline & & & mínimo & Año & Máximo & año \\
\hline Río Juncal en Juncal & $1960-2000$ & 70,06 & 42,32 & 1996 & 108,10 & 1983 \\
\hline Río Blanco en río Blanco & $1960-2000$ & 224,62 & 14,52 & 1996 & 404,92 & 1987 \\
\hline Río Colorado en Colorado & $1960-2000$ & 92,15 & 0,61 & 1996 & 237,55 & 1974 \\
\hline $\begin{array}{l}\text { Río Aconcagua en } \\
\text { Chacabuquito }\end{array}$ & $1950-2000$ & 387,20 & 142,04 & 1968 & 754,43 & 1987 \\
\hline $\begin{array}{l}\text { Río Putaendo en Resguardo } \\
\text { Los Patos }\end{array}$ & $1950-2000$ & 97,15 & 16,40 & 1996 & 251,43 & 1977 \\
\hline Río Aconcagua en San Felipe & $1961-2000$ & 278,48 & 7,14 & 1968 & 819,98 & 1992 \\
\hline
\end{tabular}

Cuadro 3

CAUDALES MEDIOS MENSUALES HISTÓRICOS, CUENCA RÍO ACONCAGUA

\begin{tabular}{|l|c|c|c|c|c|c|c|c|c|c|c|c|}
\hline \multicolumn{1}{|c|}{ Estación } & A & M & J & J & A & S & 0 & N & D & E & F & M \\
\hline $\begin{array}{l}\text { Río Juncal en Juncal } \\
\text { (1960-2000) }\end{array}$ & 4,31 & 2,80 & 2,21 & 2,05 & 1,93 & 2,19 & 4,02 & 8,00 & 11,93 & 13,07 & 10,30 & 7,25 \\
\hline $\begin{array}{l}\text { Río Blanco en río } \\
\text { Blanco (1960-2000) }\end{array}$ & 4,17 & 3,06 & 3,02 & 2,98 & 3,10 & 4,14 & 6,69 & 14,29 & 22,68 & 21,91 & 14,21 & 8,31 \\
\hline $\begin{array}{l}\text { Rio Blanco en río } \\
\text { Aconcagua (1960-2000) }\end{array}$ & 10,27 & 8,04 & 7,60 & 7,61 & 7,77 & 9,10 & 14,31 & 28,58 & 42,63 & 41,25 & 28,94 & 18,52 \\
\hline $\begin{array}{l}\text { Río Colorado en } \\
\text { Colorado (1960-2000) }\end{array}$ & 1,24 & 1,68 & 0,88 & 1,77 & 2,93 & 4,79 & 9,25 & 19,28 & 25,47 & 16,04 & 6,19 & 2,62 \\
\hline $\begin{array}{l}\text { Río Aconcagua en } \\
\text { Chacabuquito (1950- } \\
\text { 2000) }\end{array}$ & 15,8 & 13,5 & 14,2 & 14,9 & 16,0 & 21,0 & 31,4 & 58,5 & 77,5 & 60,5 & 39,1 & 24,7 \\
\hline $\begin{array}{l}\text { Río Putaendo en } \\
\text { Resguardo Los Patos } \\
\text { (1950-2000) }\end{array}$ & 3,11 & 3,10 & 3,72 & 4,67 & 6,43 & 7,47 & 11,31 & 18,12 & 18,50 & 10,98 & 5,76 & 4,00 \\
\hline $\begin{array}{l}\text { Río Aconcagua en San } \\
\text { Felipe (1961-2000) }\end{array}$ & 10,42 & 10,16 & 14,15 & 18,08 & 17,28 & 13,74 & 17,24 & 42,08 & 56,56 & 42,31 & 22,81 & 13,65 \\
\hline $\begin{array}{l}\text { Estero Catemu en } \\
\text { Puente Santa Rosa } \\
\text { (1970-2000) }\end{array}$ & 0,91 & 0,93 & 0,87 & 1,10 & 0,93 & 0,59 & 1,23 & 1,46 & 1,41 & 1,40 & 1,15 & 0,93 \\
\hline $\begin{array}{l}\text { Río Aconcagua en } \\
\text { Romeral (1961-1990) }\end{array}$ & 19,7 & 25,0 & 26,7 & 32,9 & 29,8 & 23,2 & 28,8 & 58,6 & 86,7 & 58,6 & 20,0 & 19,0 \\
\hline
\end{tabular}


Cuadro 4

CAUDALES MEDIOS MENSUALES, MÍNIMOS Y MÁXIMOS HISTÓRICOS, CUENCA RÍO ACONCAGUA

\begin{tabular}{|c|c|c|c|c|c|c|}
\hline \multirow{2}{*}{ Estación } & \multirow{2}{*}{ Serie } & \multirow{2}{*}{$\begin{array}{l}\text { Promedio } \\
\left(\mathrm{m}^{3} / \mathrm{s}\right)\end{array}$} & \multicolumn{4}{|c|}{ Caudal medio mensual $\left(\mathrm{m}^{3} / \mathrm{s}\right)$} \\
\hline & & & mínimo & Fecha & máximo & fecha \\
\hline Río Juncal en Juncal & $1960-2000$ & 5,84 & 1,31 & Julio 1969 & 30,03 & Enero 1979 \\
\hline $\begin{array}{l}\text { Rio Blanco en río } \\
\text { Blanco }\end{array}$ & $1960-2000$ & 18,72 & 0,83 & May-Jun 1996 & 92,33 & Enero 1973 \\
\hline $\begin{array}{l}\text { Río Colorado en } \\
\text { Colorado }\end{array}$ & $1960-2000$ & 7,68 & 0,01 & Varios meses* & 101,22 & Diciembre 1972 \\
\hline $\begin{array}{l}\text { Río Aconcagua en } \\
\text { Chacabuquito }\end{array}$ & $1950-2000$ & 32,27 & 4,88 & Julio 1969 & 190,74 & Diciembre 1972 \\
\hline $\begin{array}{l}\text { Río Putaendo en } \\
\text { Resguardo Los Patos }\end{array}$ & $1950-2000$ & 8,10 & 0,64 & Marzo 1997 & 67,07 & Diciembre 1953 \\
\hline $\begin{array}{l}\text { Río Aconcagua en San } \\
\text { Felipe }\end{array}$ & $1961-2000$ & 23,21 & 0,01 & Junio 1971 & 149,33 & Diciembre 1972 \\
\hline $\begin{array}{l}\text { Estero Catemu en } \\
\text { Puente Santa Rosa }\end{array}$ & $1970-2000$ & 1,07 & 0,11 & $\begin{array}{l}\text { Sep 1976; } \\
\text { agosto } 1987\end{array}$ & 12,43 & Agosto 1987 \\
\hline $\begin{array}{l}\text { Río Aconcagua en } \\
\text { Romeral }\end{array}$ & $1961-1990$ & 35,7 & 0,0 & Diciembre 1981 & 223,2 & Diciembre 1978 \\
\hline $\begin{array}{l}\text { Río Aconcagua en } \\
\text { Puente Colmo }\end{array}$ & 1997-2002 & 27,03 & 0,03 & Abril 1997 & 156,7 & Diciembre 1997 \\
\hline
\end{tabular}

* Julio-agosto 1964; Mayo a octubre 1968; abril a agosto 1969; mayo-junio 1971; febrero 1997.

Cuadro 5

CAUDAL PROMEDIO ANUAL (M³/S) SEGÚN DÉCADA PARA ESTACIONES FLUVIOMÉTRICAS DEL RÍO ACONCAGUA

\begin{tabular}{|l|c|c|c|c|c|}
\hline \multicolumn{1}{|c|}{ Estación } & $\begin{array}{c}\text { Caudal } \\
\text { promedio } \\
\text { anual serie }\end{array}$ & $\mathbf{1 9 6 1 - 1 9 7 0}$ & $\mathbf{1 9 7 1 - 1 9 8 0}$ & $\mathbf{1 9 8 1 - 1 9 9 0}$ & $\mathbf{1 9 9 1 - 2 0 0 0}$ \\
\hline Río Juncal en Juncal & 70,06 & 63,14 & 78,98 & 78,58 & 60,23 \\
\hline Río Blanco en río Blanco & 108,55 & 102,81 & 136,13 & 138,36 & 57,48 \\
\hline Río Blanco en río Aconcagua & 224,62 & 217,82 & 258,93 & 295,05 & 127,37 \\
\hline Río Colorado en Colorado & 92,15 & 67,51 & 130,15 & 114,89 & 58,52 \\
\hline Río Aconcagua en Chacabuquito & 387,20 & 320,81 & 438,70 & 485,28 & 349,32 \\
\hline Río Putaendo en Resguardo Los Patos & 97,15 & 77,18 & 105,33 & 123,07 & 82,97 \\
\hline Río Aconcagua en San Felipe & 278,48 & 157,80 & 258,14 & 371,81 & 326,17 \\
\hline Promedio & $\mathbf{1 7 9 , 7 4}$ & $\mathbf{1 7 2 , 3 8}$ & $\mathbf{2 0 9 , 5 7}$ & $\mathbf{2 5 9 , 2 0}$ & $\mathbf{1 4 5 , 2 6}$ \\
\hline
\end{tabular}


Cuadro 6

CAUDAL MEDIO MENSUAL (M³/S) SEGÚN DÉCADA PARA ESTACIONES FLUVIOMÉTRICAS DEL RÍO ACONCAGUA

\begin{tabular}{|l|c|c|c|c|c|c|}
\hline \multirow{2}{*}{ Estación } & \multirow{2}{*}{$\begin{array}{c}\text { Caudal } \\
\text { medio }\end{array}$} & \multicolumn{5}{c|}{ Caudal medio mensual según década $\left(\mathbf{m}^{3} / \mathrm{s}\right)$} \\
\cline { 5 - 7 } & serie & $\mathbf{1 9 5 0 - 1 9 6 0}$ & $\mathbf{1 9 6 1 - 1 9 7 0}$ & $\mathbf{1 9 7 1 - 1 9 8 0}$ & $\mathbf{1 9 8 1 - 1 9 9 0}$ & $\mathbf{1 9 9 1 - 2 0 0 0}$ \\
\hline Río Juncal en Juncal & 5,84 & - & 5,30 & 5,58 & 6,55 & 5,02 \\
\hline Río Blanco en río Blanco & 9,05 & - & 8,57 & 11,34 & 11,53 & 4,79 \\
\hline Rio Blanco en río Aconcagua & 18,72 & - & 18,15 & 21,58 & 24,59 & 10,61 \\
\hline Río Colorado en Colorado & 7,68 & - & 5,63 & 10,81 & 9,57 & 4,88 \\
\hline $\begin{array}{l}\text { Río Aconcagua en } \\
\text { Chacabuquito }\end{array}$ & 32,27 & 28,83 & 26,73 & 36,56 & 40,44 & 29,11 \\
\hline $\begin{array}{l}\text { Río Putaendo en Resguardo } \\
\text { Los Patos }\end{array}$ & 8,10 & 8,10 & 6,43 & 8,78 & 10,26 & 6,91 \\
\hline Río Aconcagua en San Felipe & 23,21 & - & 13,15 & 21,51 & 30,98 & 27,18 \\
\hline $\begin{array}{l}\text { Estero Catemu en Puente } \\
\text { Santa Rosa }\end{array}$ & 1,07 & - & - & 0,80 & 1,43 & 1,02 \\
\hline Río Aconcagua en Romeral & 35,7 & - & 25,20 & 33,1 & 49,0 & - \\
\hline Promedio & $\mathbf{1 6 , 5 7}$ & $\mathbf{1 8 , 4 7}$ & $\mathbf{1 4 , 3 7}$ & $\mathbf{1 7 , 3 4}$ & $\mathbf{2 1 , 6 0}$ & $\mathbf{1 2 , 1 0}$ \\
\hline
\end{tabular}

1991-2000 con caudales mensuales máximos invernales en junio (año 1986) y mayo (1993) respectivamente. Los caudales mensuales máximos según décadas ocurrieron en diciembre de $1972\left(190 \mathrm{~m}^{3} / \mathrm{s}\right), 1981\left(180 \mathrm{~m}^{3} / \mathrm{s}\right), 1982\left(180 \mathrm{~m}^{3} / \mathrm{s}\right), 1953\left(177 \mathrm{~m}^{3} / \mathrm{s}\right) 1963\left(143 \mathrm{~m}^{3} / \mathrm{s}\right)$ y enero de $1998\left(145 \mathrm{~m}^{3} / \mathrm{s}\right)$. En la estación San Felipe (curso medio de la cuenca), las variaciones estacionales e interanuales en el caudal medio mensual fueron marcadas, especialmente en el decenio 1991-2000.

El comportamiento de los caudales medios mensuales, permitió distinguir dos regímenes hidrológicos: El primero, corresponde al curso superior del río Aconcagua, desde el nacimiento de los principales afluentes en la cordillera de Los Andes hasta la entrada del río Aconcagua en el valle, con un régimen principalmente nival. Las estaciones fluviométricas de río Juncal, Río Blanco, río Colorado y Chacabuquito fueron representativas de este sector con caudales medios mensuales máximos en diciembre del orden de los $58,5 \mathrm{~m}^{3} / \mathrm{s}$ en Chacabuquito (Fig. 2A). El segundo, corresponde al curso medio-inferior del río Aconcagua, donde el régimen es nivo-pluvial donde las estaciones fluviométricas representativas correspondieron a San Felipe y Romeral (Fig. 2B). La década que registró el mayor caudal en la cuenca del Aconcagua fue 1981-1990 y aquella que señaló el menor caudal fue la de 1961-1970 donde los valores fueron inferiores al promedio anual de la serie histórica (Fig. 3).

En el caso de los caudales medios mensuales extraordinarios, estos se registraron en los meses estivales de los años 1972, 1973, 1978, 1979, 1987 y 1997 coincidentes con eventos El Niño. Los caudales anuales mínimos fueron coincidentes con aquellos años en los cuales se produjeron las mayores sequías en el país, principalmente 1968. Los años 1996, 1998 y 
Figura 2

CAUDALES MEDIOS MENSUALES DE LA CUENCA DEL RIOO ACONCAGUA
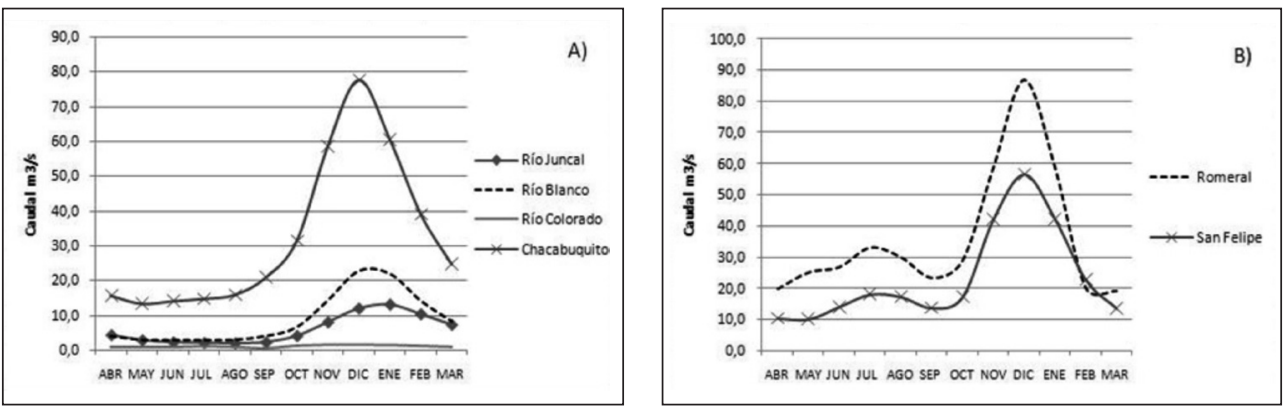

Figura 3

CAUDALES MEDIOS ANUALES DE LA CUENCA DEL RÍO ACONCAGUA, EN DIFERENTES DÉCADAS

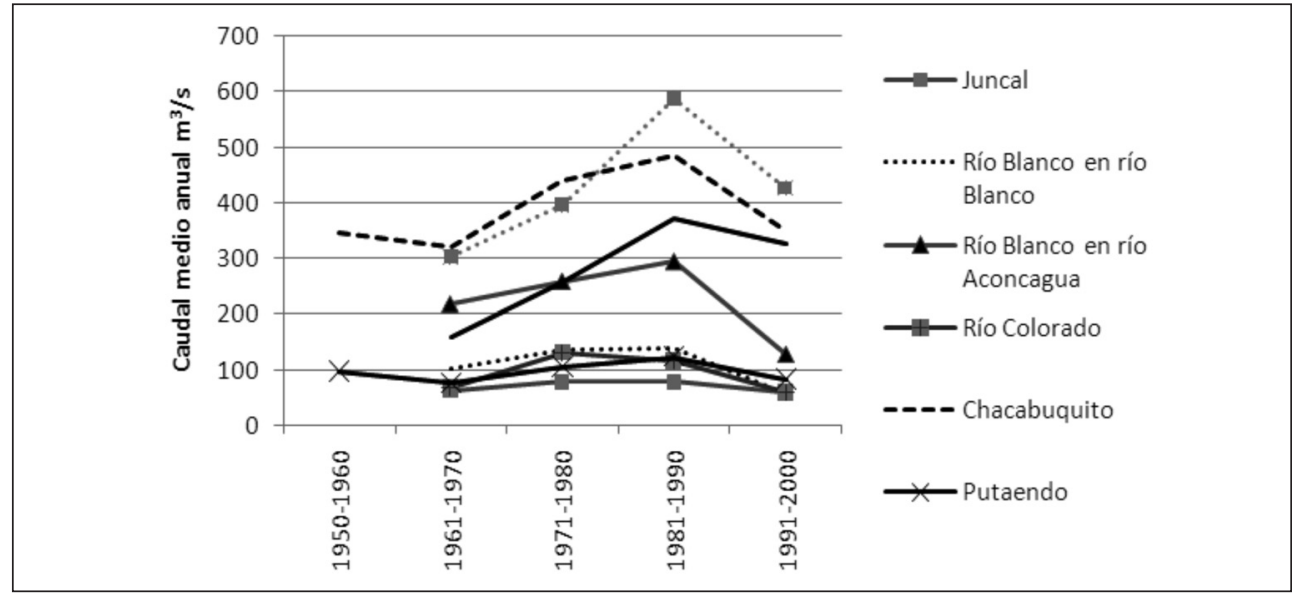

1999 fueron coincidentes con eventos La Niña. Los caudales medios mensuales máximos se registraron en su totalidad durante el período de deshielos. Los caudales mínimos mostraron una amplia fluctuación estacional durante los años 1969, 1971, 1976, 1981, 1987, 1996 y 1997.

El ajuste de los caudales medios mensuales registrados para cada estación frente a las distintas distribuciones de probabilidad (Cuadro 7), estableció que la mayoría de las estaciones fueron correctamente modeladas por la distribución Pearson III. Se destaca que los años de mayor caudal se asociaron a un ciclo de años seguidos con esta misma característica. De hecho, mientras en las estaciones de Chacabuquito, Río Blanco en Aconcagua, Río Blanco en Río blanco, Colorado y San Felipe, se registraron los máximos en el período 1972-1973, en el caso de las estaciones Juncal y Putaendo, esto ocurrió en el período 1977-1979. Utilizando las mismas distribuciones normalizadas para períodos seculares, los mayores caudales 
medios mensuales registrados presentaron una recurrencia mayor a 90 años. Específicamente, el caso del registro de 1972 fluctuó entre 96 y 99 años.

Cuadro 7

AJUSTE DE DISTRIBUCIONES DE PROBABILIDAD DE LOS REGISTROS DE LAS ESTACIONES SELECCIONADAS DE ACUERDO AL TEST DE KOLMOGOROV-SMIRNOV

\begin{tabular}{|c|c|c|c|c|}
\hline Estación/distribución & Normal & Lognormal & Gumbel & Pearson III \\
\hline Juncal & 0.17 & 0.13 & $0.47^{*}$ & 0.42 \\
\hline Río Blanco & $0.95^{*}$ & 0.06 & 0.69 & 0.83 \\
\hline Río Colorado & 0.46 & 0.34 & 0.90 & $0.95^{*}$ \\
\hline Chacabuquito & 0.24 & 0.68 & 0.68 & $0.80^{*}$ \\
\hline Putaendo & 0.23 & 0.82 & 0.82 & $0.99^{*}$ \\
\hline San Felipe & $0.96^{*}$ & 0.06 & 0.69 & 0.83 \\
\hline Río Blanco en Río Blanco & 0.46 & 0.33 & 0.90 & $0.95^{*}$ \\
\hline
\end{tabular}

El valor corresponde al p value. Asterisco indica distribución seleccionada.

Se destaca que para décadas con caudal normal a bajo, se registró una fuerte influencia de años calificados como secos, tales como 1968 y 1996 caracterizados por sequías extremas, así también los años anteriores a estos. Del comportamiento anterior se presentó por lo tanto, una tendencia general a la disminución del caudal a través del tiempo. De acuerdo a la Fig. 4, tal tendencia es generalizada a todas las estaciones fluviométricas de la cuenca, siendo las estaciones con mayor caudal las localizadas en el curso inferior del río como Chacabuquito y San Felipe. La estación Río Aconcagua en río Blanco registró un descenso brusco en las dos últimas décadas.

Las siguientes características hidrológicas han sido determinadas para cada estación fluviométrica:

a) Estación río Juncal en Juncal: en el período 1961-2000 esta estación registró una tendencia a la disminución del caudal. El caudal promedio anual fue de 70,06 $\mathrm{m}^{3} / \mathrm{s}$ mientras que el medio mensual de $5,84 \mathrm{~m}^{3} / \mathrm{s}$. Según el ajuste lineal de la Fig. 5A, la tendencia a la disminución supone una reducción de $0,84 \mathrm{~m}^{3} / \mathrm{s}$ por década.

b) Estación río Blanco en Río Blanco: en el período 1961-2000 esta estación registró una tendencia brusca a la disminución del caudal principalmente desde 1990 en adelante. El caudal promedio anual fue de $108,5 \mathrm{~m}^{3} / \mathrm{s}$ mientras que el medio mensual de $9,05 \mathrm{~m}^{3} / \mathrm{s}$. Según el ajuste lineal de la Fig. 5B, la tendencia a la disminución supone una reducción de $14 \mathrm{~m}^{3} / \mathrm{s}$ por década.

c) Estación río Aconcagua en Río Blanco: en el período 1961-2000 esta estación registró una tendencia brusca a la disminución del caudal principalmente desde 1990 en adelante. El caudal promedio anual fue de 224,62 $\mathrm{m}^{3} / \mathrm{s}$ mientras que el medio mensual de $18,72 \mathrm{~m}^{3} / \mathrm{s}$. Según el ajuste lineal de la Fig. 5C, la tendencia a la disminución supone una reducción de $25,5 \mathrm{~m}^{3} / \mathrm{s}$ por década. 
Figura 4

TENDENCIA LINEAL DE LOS CAUDALES REGISTRADOS EN CADA ESTACIÓN PARA VERANO (A), INVIERNO (B) YAÑO (C)
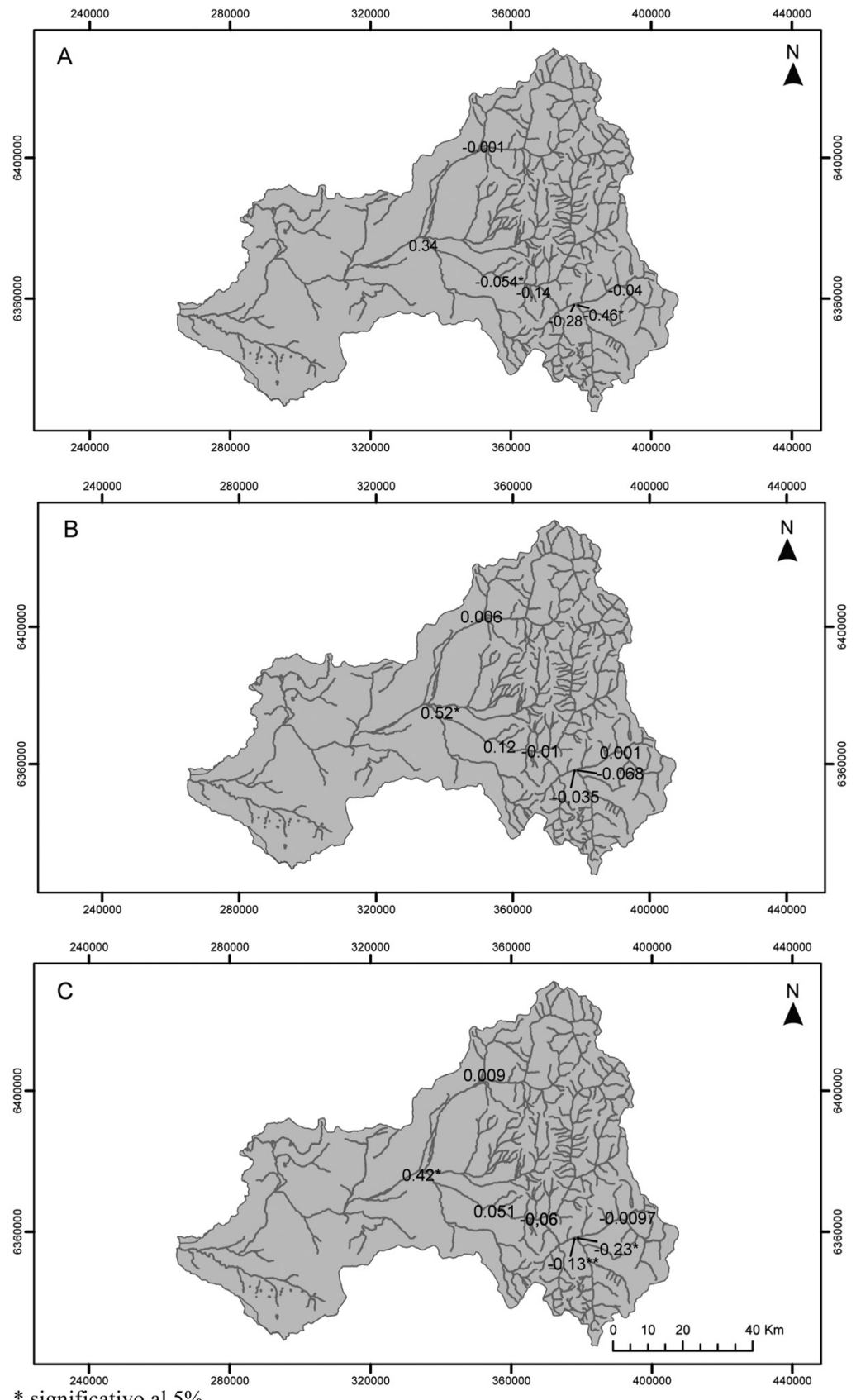

* significativo al $5 \%$

$* *$ significativo al $1 \%$ 
d) Estación río Colorado en Río Colorado: en el período 1961-2000 esta estación registró una tendencia también brusca a la disminución del caudal principalmente desde 1988 en adelante. El caudal promedio anual fue de $92,15 \mathrm{~m}^{3} / \mathrm{s}$ mientras que el medio mensual de $7,68 \mathrm{~m}^{3} / \mathrm{s}$. Según el ajuste lineal de la Fig. 5D, la tendencia a la disminución supone una reducción de $5,6 \mathrm{~m}^{3} / \mathrm{s}$ por década.

e) Estación río Aconcagua en Chacabuquito: en el período 1950-2000 esta estación registró una leve tendencia al aumento del caudal principalmente desde 1981 en adelante, con algunas interrupciones en los años 1990 y 1990 en que se registró bajo caudal. El caudal promedio anual fue de $387,20 \mathrm{~m}^{3} / \mathrm{s}$ mientras que el medio mensual

Figura 5

TENDENCIAS EN CAUDALES MEDIOS ANUALES EN LA CUENCA DEL RÍO ACONCAGUA

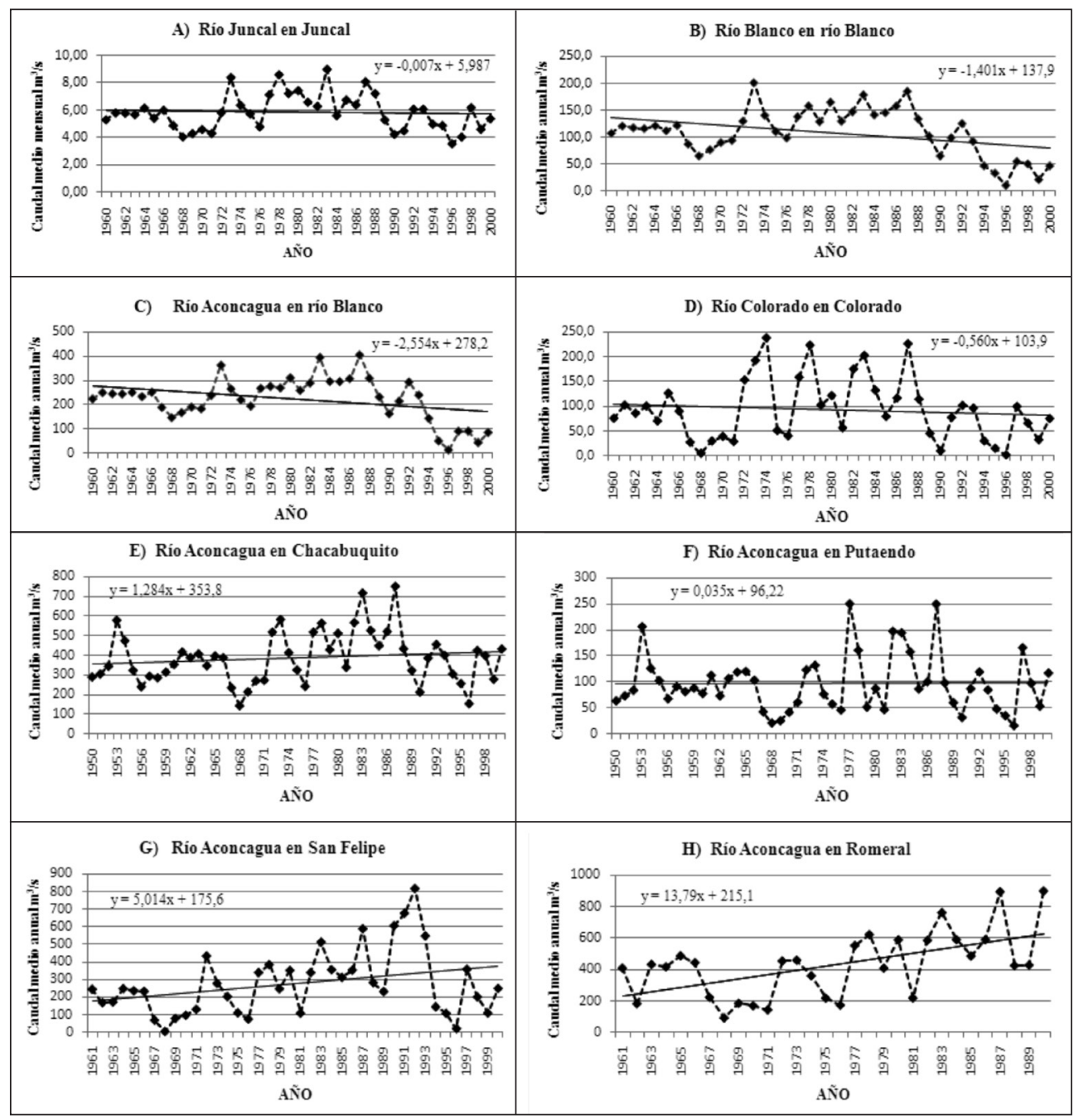


de $32,27 \mathrm{~m}^{3} / \mathrm{s}$. En esta estación se registran los mayores caudales de la cuenca después de la estación de Romeral. Según el ajuste lineal de la Fig. 5E, se presenta una leve tendencia que supone un aumento de $12,8 \mathrm{~m}^{3} / \mathrm{s}$ por década.

f) Estación río Aconcagua en Putaendo: en el período 1950-2000 esta estación registró una escasa tendencia al aumento de los caudales. El caudal promedio anual fue de $97,15 \mathrm{~m}^{3} / \mathrm{s}$ mientras que el medio mensual de $8,10 \mathrm{~m}^{3} / \mathrm{s}$. Según el ajuste lineal de la Fig. $5 \mathrm{~F}$, la tendencia a la disminución supone una reducción de $0,35 \mathrm{~m}^{3} / \mathrm{s}$ por década.

g) Estación río Aconcagua San Felipe: en el período 1961-2000 esta estación registró una escasa tendencia al aumento de los caudales. El caudal promedio anual fue de $278,48 \mathrm{~m}^{3} / \mathrm{s}$ mientras que el medio mensual de $23,21 \mathrm{~m}^{3} / \mathrm{s}$. Según el ajuste lineal de la Fig. 5G, la tendencia al aumento supone un aumento de $50,1 \mathrm{~m}^{3} / \mathrm{s}$ por década.

h) Estación río Aconcagua en Romeral: en el período 1961-1990 esta estación registró una escasa tendencia al aumento de los caudales. El caudal promedio anual fue de $428,9 \mathrm{~m}^{3} / \mathrm{s}$ mientras que el medio mensual de $35,7 \mathrm{~m}^{3} / \mathrm{s}$. Según el ajuste lineal de la Fig. $5 \mathrm{H}$, se registra una importante tendencia al aumento que supone un aumento de $137,9 \mathrm{~m}^{3} / \mathrm{s}$ por década. Sin embargo, si se considera una serie mayor pero discontinua (1961-1990; 1995; 1999-2006) la tendencia se mantuvo pero algo menor involucrando $51,6 \mathrm{~m}^{3} / \mathrm{s}$ por década.

De acuerdo con el análisis anterior, en la cuenca del río Aconcagua es posible establecer que el $50 \%$ de las estaciones fluviométricas se encuentran en situación de déficit hídrico mientras que el $50 \%$ restante presenta tendencia al superávit. En el primer caso, se encuentran las estaciones de Juncal, Río Blanco, Río Colorado y Putaendo. Aquellas que presentaron una leve tendencia al aumento del caudal son Chacabuquito, San Felipe, Catemu

Figura 6

CURVA DE TENDENCIA PARA CAUDALES MEDIOS ANUALES, CUENCA DEL RIOO ACONCAGUA*

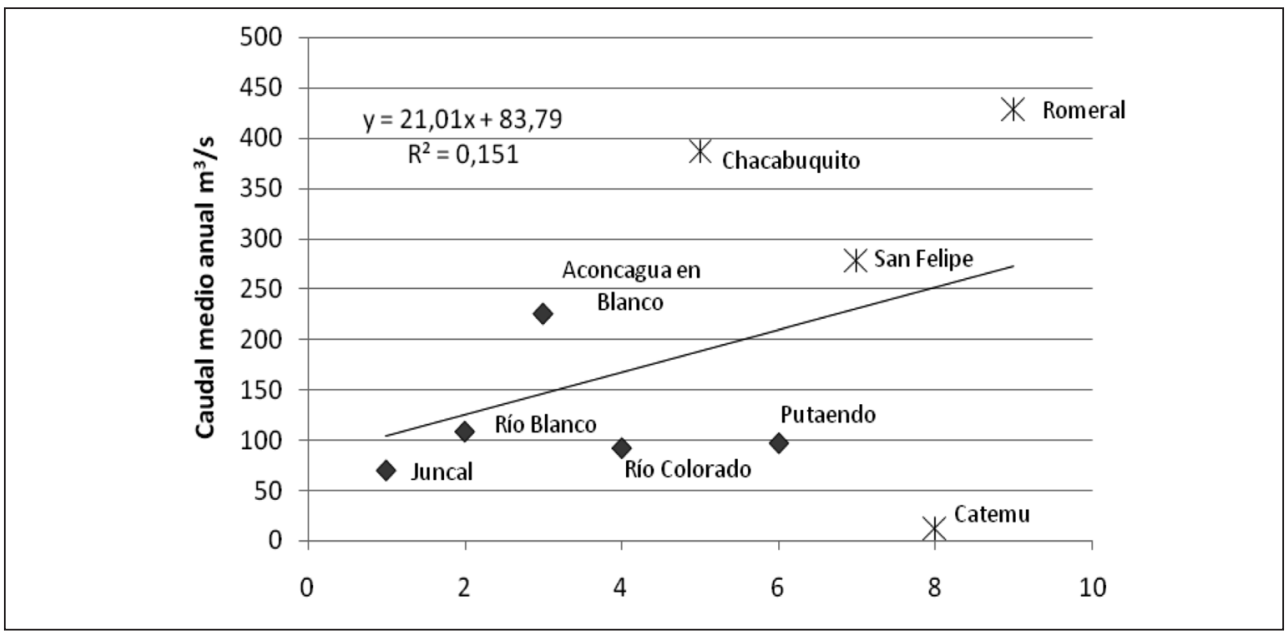

* Los rombos indican estaciones en déficit hídrico y los asteriscos estaciones en superávit. 
y Romeral. La mayor tendencia al déficit en el caudal respecto al promedio histórico se registró en la estación de Río Blanco con 11,3\% y Río Colorado con 6\%. El aumento de caudal más relevante se presentó en las estaciones de Romeral con 32,1\% y San Felipe con 17,9\%. De acuerdo con la Fig. 6, todas las estaciones del curso superior del río Aconcagua se presentaron con déficit hídrico mientras que a partir del curso medio a inferior las estaciones se registraron con una leve tendencia a la excedencia (símbolos en asterisco), especialmente la estación de Romeral. La curva de tendencia indica que existe escasa asociación entre el aumento de los caudales y la distancia entre el curso superior e inferior del río $\left(R^{2}=0,15\right)$, aunque es posible establecer que las estaciones que se encuentran más alejadas de la línea de ajuste son aquellas localizadas en el curso medio-inferior.

\section{Caudales y eventos ENSO}

En el caso de la comparación entre el índice TSM y los caudales para el mismo período de tiempo (Cuadro 8), se observó una correlación positiva y significativa. Esto significa que un aumento de los caudales de verano está directamente relacionado con un aumento de la temperatura superficial del mar producto del ENSO. En ese sentido, son notables las correlaciones de las estaciones Chacabuquito y San Felipe, con valores superiores de 0,5 a 1\% de significancia. Para caudales de invierno, sólo la estación de San Felipe presentó una correlación significativa. Adicionalmente, se observó que hay tres estaciones donde no hubo correlación. Para el caso de la correlación entre la TSM y los caudales de la estación siguiente a esta medición, sólo se encontraron correlaciones significativas con las estaciones del curso medio del río, donde la más alta y significativa se observó entre el ENSO de invierno y el caudal de verano consecutivo en Chacabuquito. No se encontró correlación significativa entre el valor de las estaciones de invierno y su verano antecedente (Cuadros 9 y 10).

Cuadro 8

CORRELACIONES TSM - CAUDALES DURANTE LA MISMA ESTACIÓN

\begin{tabular}{|l|l|l|l|l|}
\hline & \multicolumn{2}{c|}{ DEF } & \multicolumn{2}{c|}{ JJA } \\
\hline \multicolumn{1}{|c|}{ Estación } & \multicolumn{1}{c|}{ R } & \multicolumn{1}{c|}{$\mathbf{R}^{2}$} & \multicolumn{1}{c|}{ R } & \multicolumn{1}{c|}{$\mathbf{R}^{2}$} \\
\hline Chacabuquito & $0.50^{* *}$ & 0.25 & $0.50^{*}$ & 0.25 \\
\hline Río Blanco & $0.39^{*}$ & 0.16 & $0.32^{*}$ & 0.1 \\
\hline San Felipe & $0.53^{* *}$ & 0.28 & $0.52^{* *}$ & 0.28 \\
\hline Río Blanco RBLAN & $0.34^{*}$ & 0.12 & 0.26 & 0.06 \\
\hline Colorado & $0.49^{* *}$ & 0.24 & 0.03 & 0.001 \\
\hline Juncal & $0.45^{* *}$ & 0.21 & 0.03 & 0.001 \\
\hline Putaendo & $0.49^{* *}$ & 0.24 & $0.36^{*}$ & 0.12 \\
\hline
\end{tabular}

* significativo al $5 \%$

** significativo al $1 \%$ 
Cuadro 9

CORRELACIONES TSM VERANO - CAUDALES INVIERNO CONSECUTIVO

\begin{tabular}{|c|c|c|}
\hline & \multicolumn{2}{|c|}{ JJA } \\
\hline Estación & $\mathbf{R}$ & $\mathbf{R}^{2}$ \\
\hline Chacabuquito & 0.18 & 0.03 \\
\hline Río Blanco & 0.18 & 0.03 \\
\hline San Felipe & 0.31 & 0.09 \\
\hline Río Blanco RBLAN & 0.11 & 0.01 \\
\hline Colorado & -0.17 & 0.03 \\
\hline Juncal & $0.36 *$ & 0.13 \\
\hline Putaendo & 0.2 & 0.03 \\
\hline
\end{tabular}

* significativo al $5 \%$

** significativo al $1 \%$

Cuadro 10

CORRELACIONES TSM INVIERNO - CAUDALES VERANO CONSECUTIVO

\begin{tabular}{|c|c|c|}
\hline & \multicolumn{2}{|c|}{ DEF } \\
\hline Estación & $\mathbf{R}$ & $\mathbf{R}^{2}$ \\
\hline Chacabuquito & $0.34 * *$ & 0.11 \\
\hline Río Blanco & 0.24 & 0.23 \\
\hline San Felipe & $0.36^{*}$ & 0.12 \\
\hline Río Blanco RBLAN & 0.18 & 0.68 \\
\hline Colorado & $0.32 *$ & 0.01 \\
\hline Juncal & 0.27 & 0.07 \\
\hline Putaendo & $0.37 *$ & 0.13 \\
\hline
\end{tabular}

* significativo al $5 \%$

$* *$ significativo al $1 \%$

\section{DISCUSIÓN}

Autores que han analizado las influencias de ENOS (El Niño, Anti-Niño y año normal) sobre las respuestas en ríos sudamericanos (Waylen y Caviedes, 1990; Caviedes y Waylen, 1998), han determinado un comportamiento estacional de los caudales mensuales en ríos de Chile central, los cuales se incrementan como resultado de la presencia de eventos de El Niño (EN) o fase cálida y disminuyen en condiciones de Anti-Niño (AN) o fase fría, estableciendo además que el río Aconcagua constituye el mejor ejemplo de este tipo de comportamiento. En esta investigación, se obtuvieron resultados similares, porque la respuesta de este sistema fluvial frente a la fase fría ENSO es una abrupta disminución de los caudales aún interca- 
lados en años clasificados como normales (años 1962 y 1981), aunque el comportamiento general es una disminución gradual de los caudales como ocurrió en las grandes sequías hidrológicas históricas de la región de 1968 y 1996. Para este último caso, Castillo (2003) verificó a través de anomalías de temperatura superficial del mar (TSM) para la región Niño 3, dos períodos La Niña: julio de 1995 a diciembre de 1996 y septiembre de 1998 a mayo de 2000. En 1997-1998 constituye en último gran El Niño. Las estaciones fluviométricas que mejor reaccionan a las fases cálidas y frías ENOS son Chacabuquito y San Felipe en el curso medio-inferior de la cuenca, las cuales además presentan altas correlaciones con los índices TSM en especial durante el verano.

Durante los últimos 40 años, solo la década 1981-1990 supera el promedio histórico para cada estación fluviométrica. Esto podría relacionarse con el predominio de fases cálidas ENOS que fueron más recurrentes y fuertes que en las décadas anteriores, destacando el de 1982-83. A partir de 1990, todas las estaciones presentaron un marcado descenso de los caudales. Sin embargo, no toda la cuenca registra un comportamiento similar ya que las estaciones localizadas en el curso superior han establecido un importante déficit de los caudales. De este modo se determinó que las estaciones Juncal, Río Aconcagua en río Blanco, Río Blanco, Río Colorado y Putaendo se encontraron en déficit hídrico de hasta un 11,3\% respecto al caudal medio anual histórico. Sin embargo, las estaciones localizadas en el curso medio e inferior como Chacabuquito, San Felipe, Catemu y Romeral registraron una tendencia al aumento pudiendo llegar al 32,1\% en Romeral.

De cualquier forma, la tendencia positiva de las estaciones fluviométricas del curso inferior del río Aconcagua no indica que se está frente a un escenario de abundancia del recurso hídrico ya que ésta puede ser opuesta si se incluye en el ajuste de tendencia la década de 2000 que se ha presentado menos abundante que la anterior. Para el período 1999-2006, la estación de Romeral registró un caudal medio anual de 392,1 m³/s inferior al promedio histórico mientras que entre 1997-2002 la estación de Puente Colmo (actualmente deshabilitada) registró un promedio de solo $132 \mathrm{~m}^{3} / \mathrm{s}$. Si se compara igual período en la estación de Romeral se obtienen $400,6 \mathrm{~m}^{3} / \mathrm{s}$ es decir un valor cercano al promedio histórico de $428,8 \mathrm{~m}^{3} / \mathrm{s}$. Lo anterior indica que en el curso medio del río Aconcagua existe un excedente del recurso hídrico debido al aporte de aguas subterráneas al flujo libre del río, pero este excedente no llega el curso inferior del río debido a que probablemente se consume.

La tendencia negativa de los caudales fue recientemente establecida por Givovich (2006) al analizar su comportamiento mensual en subcuencas nacientes de los grandes ríos Maipo y Aconcagua en relación con posibles efectos del cambio climático. Utilizando las mismas estaciones fluviométricas que en esta investigación, el autor encontró que en las cuencas de los ríos Juncal, Colorado y Blanco existe una disminución de los caudales para el período 1950-1999 que fluctúa entre 10,4\%; 20,2\% y 12,1\% respectivamente para los últimos 10 años. Al contrario, para la estación de Chacabuquito encontró una tendencia al alza con una variación de 1,7\% para los últimos 10 años. Analizando las temperaturas y las precipitaciones, determinó un incremento de $0,57^{\circ} \mathrm{C}$ en la temperatura media anual entre 1973 y 2004 para la banda de los $2.000 \mathrm{~m}$ de altura, especialmente durante los meses julio y agosto en los cuales se registran los principales aportes nival y pluvial. El autor asoció la tendencia al aumento de los caudales encontrada en las estaciones de aforo de Chacabuquito (Río Aconcagua) y El Manzano (río Maipo) al cambio climático que estaría afectando el derre- 
timiento de nieves a esa altura, debido al aumento de las temperaturas. Al respecto, se ha establecido una relación entre el aumento de las temperaturas y las precipitaciones con el proceso de deglaciación que estaría afectando los glaciares de la zona central de Chile y Patagonia (Rivera y Cassasa, 1999; Rivera et al., 2000). Por otro lado, Rivera et al., (2002) investigando el comportamiento histórico de 95 glaciares chilenos estableció una fuerte tendencia al retroceso frontal de los glaciares para el $88 \%$ de estos. Para los glaciares del campo de hielo de Patagonia sur $\left(48^{\circ}-52^{\circ} \mathrm{S}\right)$, Rivera y Cassasa (2004) encontraron igual tendencia desde 1945 como resultado del aumento de las temperaturas que influencian las altas tasas de ablación.

El estudio del balance de masa en algunos glaciares como Echaurren Norte (río Maipo) presentan una fuerte variabilidad y la tendencia se relacionaría más con los ciclos ENOS que con el cambio climático propiamente tal. Por otro lado, también se ha establecido que los glaciares pueden mostrar distintos comportamientos frente a un mismo escenario tanto de temperaturas como de precipitaciones, en consecuencia, los tiempos de respuesta son diferentes (Fernández et al., 2006). Aunque la relación entre ablación en glaciares andinos y la fase cálida ENOS parece más clara (Escobar y Aceituno, 1998; Prieto et al., 2001), la relación en fase fría ENOS y el proceso de acumulación de la nieve superior a la media en Los Andes centrales no está tan clara para la región Niño 3.4 y parece ser mas compleja (Masiokas et al., 2006).

En el contexto mundial, el aumento de los caudales por el incremento del aporte pluvial durante fases cálidas ENOS es un fenómeno conocido aunque no un patrón lineal. Para cuencas de Sudamérica, Waylen y Caviedes (1990) encontraron que durante fases cálidas ENOS, algunos ríos de Ecuador y Perú reaccionan inmediatamente al inicio del verano austral, mientras que otros como los del noroeste árido de Argentina o aquellos interiores de la sierra peruana, reaccionan de manera opuesta, con descargas bajas durante los veranos ENOS. Por otro lado, ríos de la Patagonia del sur del continente son indiferentes o tienen una baja sensibilidad a las fases ENOS.

La variabilidad de las estaciones fluviométricas en la cuenca del río Aconcagua respecto a las correlaciones TSM-caudal resultó importante. Las mejores asociaciones (positivas) se registraron en las estaciones Chacabuquito y San Felipe localizadas en el curso medio de la cuenca. Esta variabilidad ya ha sido descrita por algunos autores para ríos sudamericanos (Gutiérrez et al., 1998; Lagos et al., 2008); así como también se ha indicado una mayor asociación entre evento cálido y aumento del caudal en el período de descarga inmediato (Norte et al., 1998). Esta variabilidad está relacionada con los distintos modos ENOS, como respuesta a los mecanismos de teleconexión océano-atmósfera en el Pacífico. El nuevo modo de El Niño denominado El Niño Modoki, El Niño del Pacífico Central o Pseudo Niño (Ashok et al., 2007; Li et al., 2010; Takahashi et al., 2011) el cual registró una anomalía TSM máxima en la región del Pacífico central en 2004, describiría en parte el tradicional modo de El Niño. Sin embargo, algunos de sus efectos son diferenciales respecto al modo tradicional de El Niño y de acuerdo a algunas investigaciones presentaría en los próximos años mayor recurrencia que la fase tradicional (Ashok et al., 2007). En Australia, el Niño Modoki dependiendo de su magnitud y localización, afecta la intensidad de las precipitaciones reduciéndolas en diciembre y entre marzo a mayo, e intensificándolas entre enero y febrero, afectando el período de monzones (Taschetto y England, 2009). Aunque se ha establecido 
una independencia lineal entre ambos modos de El Niño y por lo tanto se tratarían de eventos diferentes (Li et al., 2010) es indudable que la teleconexión asociada a estos mecanismos pueden hacer más compleja la explicación sobre la variabilidad climática en la Región Niño 3.4, principalmente es su relación con el comportamiento de las precipitaciones y sus efectos en la descarga de los ríos andinos.

Como la mayoría de las cuencas andinas de Chile central, la cuenca del río Aconcagua está altamente antopizada en su curso medio e inferior, mientras que en su curso superior se desarrollan actividades de turismo invernal con infraestructura asociada. Algunos de los efectos de la antropización en los glaciares de montaña que alimentan la red hídrica han sido analizados por Corripio et al., (2007) y los problemas asociados al manejo del agua por Ribbe et al., (2002) y Ribbe y Gaese (2004). La demanda del agua para fines industriales, agrícolas o urbanos no ha sido analizada en un contexto de manejo integrado de los recursos hídricos como se ha propuesto por ejemplo para la cuenca del río Maipo (Rosegrant et al., 2000) o en perspectivas multi-institucionales e interdisciplinares para adaptación de los usos a la variabilidad climática (Martínez et al., 2008). Estos usos o demandas hídricas afectan en diferentes escalas de tiempo, la respuesta de los caudales a los fenómenos interdecadales o interanuales del clima y en el caso de la cuenca del río Aconcagua podría ser un factor importante y necesario de abordar en futuras investigaciones.

El comportamiento de los caudales en la cuenca, es un aspecto prioritario para considerar en el manejo del agua dado que desde 2002 la Dirección General de Aguas evaluó algunos sectores del acuífero con fines de sustentabilidad de derechos de agua, estableciendo que algunos sectores del curso inferior presentan una demanda mayor que la oferta de caudal sustentable. A esto se debe considerar el aumento de los derechos de agua para riego y que las cuencas localizadas al norte son afectadas por condiciones climáticas más áridas, tales como las de Copiapó y Huasco que se encuentran con graves déficit de aguas subterráneas provocadas por el uso principalmente minero.

\section{CONCLUSIONES}

Se encontró una alta variabilidad en el comportamiento histórico de los caudales para el período considerado, con fluctuaciones importantes entre las estaciones fluviométricas evaluadas y en relación con mínimos y máximos históricos, así como entre períodos estacionales. Los caudales medios anuales máximos se registraron en las estaciones Chacabuquito y San Felipe (sección media de la cuenca) con 754 (año 1987) y 819 m³/s (año 1992) respectivamente, es decir asociados a eventos El Niño. Los caudales medios anuales máximos y mínimos de todas las estaciones fluviométricas se produjeron durante años clasificados como El Niño o La Niña, estableciéndose que la cuenca es altamente sensible a los eventos ENOS. Así también, la influencia de los eventos ENOS se reflejó en aumentos o disminuciones de los caudales en años previos y consecutivos a estos eventos, principalmente para eventos La Niña.

El grado de reacción de los caudales de la cuenca a los eventos ENOS es también variable, tendiendo a reaccionar mejor frente a anomalías TSM ocurridas durante la misma estación climática. Las anomalías TSM positivas se correlacionan mejor durante la estación estival, reflejándose en un aumento de los caudales de verano. 
Dado que el $50 \%$ de las estaciones fluviométricas establecieron una tendencia al déficit hídrico (curso superior de la cuenca) y el 50\% restante registraron una tendencia al superávit (curso medio-inferior), resulta relevante para el manejo de los recursos hídricos en la cuenca, establecer a través de futuras investigaciones, el rol que está ejerciendo el uso del agua en el balance hídrico de las aguas superficiales y subterráneas de la cuenca. En este sentido, se debe destacar que la definición y el establecimiento de caudal sustentable se realizan, por lo general, a partir de registros de bioindicadores (fito y zoo) y variaciones de caudal, con los cuales es posible establecer el caudal ecológico del sistema. Este hecho unido a lo primario aún de los establecimientos de las variaciones de caudal a partir de observaciones físicas, obliga a direccionar la búsqueda de unos patrones de variación del caudal de tipo biofísico, con los cuales se podrían empezar a establecer con mayor precisión los códigos o normas de extracción y uso del agua que no comprometan en un largo plazo la sustentabilidad del recurso agua.

\section{AGRADECIMIENTOS:}

Se agradece a los revisores anónimos los valiosos aportes al trabajo. Este trabajo fue desarrollado en el contexto de la tesis doctoral «Geomorfología Dinámica del sistema costero de la bahía de Concón (Chile Central)», Martínez, C. (2009). Universitat de Barcelona.

\section{BIBLIOGRAFÍA}

ACEITUNO, P. y VIDAL, F. (1990): «Variabilidad interanual en el caudal de ríos andinos en Chile central en relación con la temperatura superficial del mar en el Pacífico ecuatorial central». Revista de la Sociedad Chilena de Ingeniería Hidráulica, no 5, pp. 7-19.

APARICIO, F. (1992): Fundamentos de Hidrología de Superficie. México. Limusa.

ASHOK, K., BEHERA, S., RAO, S., WENG, H. y YAMAGATA, T. (2007): «El Niño Modoki and its possible teleconnection». Journal of Geophysical Research, $\mathrm{n}^{\circ} 112$ (C11007), doi:10.1029/2006JC003 798.

CASTILlO, M. (2003): «Ciclos El Niño/La Niña. Período 1991-2000 y su influencia en las costas norte y central de Chile» en Anuario. Valparaíso, Ed. Servicio Hidrográfico y Oceanográfico de la Armada de Chile, Tomo 55, pp. 1-8.

CAVIEDES, C. (1967): «Las terrazas del Aconcagua inferior». Revista Geográfica de Valparaíso, vol. 1, no 1 , pp. 63-80.

CAVIEDES, C. (1972): «Geomorfología del Cuaternario del valle del Aconcagua, Chile central». Freiburger Geographische Hefte, № 11 .

CAVIEDES, C. y WAYLEN, P. (1998): «Respuesta del clima de América del Sur a las fases de ENSO». Bulletin de l'Institut Français d'Ètudes Andines, vol. 27, nº 3, pp. 613-626.

CORRIPIO, J., PURVES, R. y RIVERA, A. (2007): «Modeling climate-change impacts on mountain glaciers and water resources in the Central Dry Andes» en Darkening Peaks: Glacier Retreat, Science and Society (Orlove, B., Wiegandt, E. y Luckman, B. Eds.). USA, University of California Press, pp. 126-135.

DIRECCIÓN GENERAL DE AGUAS (2004): Evaluación de los recursos hídricos superficiales en la cuenca del río Aconcagua. S.D.T. N ${ }^{\circ}$ 165. Departamento de Administración de Recursos Hídricos, Santiago. 
DIRECCIÓN GENERAL DE AGUAS (2002): Evaluación de los recursos subterráneos de la cuenca del río Aconcagua. Departamento de Administración de Recursos Hídricos, Santiago.

ERRÁZURIZ, A., CERECEDA, P., GONZÁLEZ, J., GONZÁLEZ, M., HENRÍQUEZ, M. y RIOSECO, R. (1998): Manual de Geografía de Chile. Santiago, Andrés Bello.

ESCOBAR, F. y ACEITUNO, P. (1998): Influencia del fenómeno ENSO sobre la precipitación nival en el sector andino de Chile central durante el invierno. Bulletin de l'Institut Français d'Ètudes Andines, vol. 27, nº 3, pp. 753-759.

FERNÁNDEZ, A., RIVERA, A. y RODRIGO, C. (2006): «Variaciones Recientes de Glaciares entre $41^{\circ} \mathrm{S}$ y $49^{\circ} \mathrm{S}$ y su Relación con los Cambios Climáticos». Revista Geográfica del Instituto Panamericano de Geografía e Historia (IPGH) nº 139, pp. 39-69.

GAJARDO, R. (1994): La Vegetación Natural de Chile, Clasificación y Distribución Geográfica. CONAF. Santiago, Universitaria.

GIVOVICH, W. (2006): «Derretimiento de las nieves y recursos hídricos de la zona CentroNorte de Chile». Revista Ambiente y Desarrollo, vol. 22, nº 1, pp. 58-67.

GUTIÉRREZ, F., PIECHOTA, T. y DRACUP, J. (1998): «Conexiones entre caudales de algunos ríos de la costa norte y central del Perú y El Niño». Bulletin de l'Institut Français d'Ėtudes Andines, vol. 27, nº 3, pp. 829-838.

LAGOS, P., SILVA, Y., NICKL, E. y MOSQUERA, K. (2008): «El Niño-related precipitation variability in Perú». Advances in Geosciences, $\mathrm{n}^{\circ}$ 14, pp. 231-237.

LI, G., REN, B., YANG, CH. y ZHENG, J. (2010): «Traditional El Niño and El Niño Modoki Reviseted: Is El Niño Modoki linearly independent of tradictional El Niño?». Atmospheric and Oceanic Science Letters, vol 3, $\mathrm{n}^{\circ}$ 2, pp. 70-74.

MARTÍNEZ, A., TAKAHASHI, K., NÚÑEZ, E., SILVA, Y., TRASMONTE, G., MOSQUERA, K. y LAGOS, P. (2008): «A multi-institutional and interdisciplary approach to the assessment of vulnerability and adaptation to climate change in the Peruvian Central Andes: problems and prospects». Advances in Geosciences, $\mathrm{n}^{\circ}$ 14, 257-260.

MARTÍNEZ, C. (2009) «Geomorfología Dinámica del sistema costero de la bahía de Concón (Chile Central)». Tesis Doctoral. Departament de Geografia Fisica i AGR. Universitat de Barcelona.

NIEMEYER, H. y CERECEDA, P. (1984): Hidrografía. Colección Geografía de Chile. Tomo VIII. Santiago, Instituto Geográfico Militar.

NORTE, F., SIMONELLI, S. y HEREDIA, N. (1998): «Impacto del fenómeno ENOS en el régimen hidrometeorológico de Mendoza, Argentina». Bulletin de l'Institut Français d'Ètudes Andines, vol. 27, nº 3, 761-770.

PRIETO, R., HERRERA, H., DOUSSEL, P., GIMENO, L. y P. RIBERA. (2001): «Interanual oscillations and trend of snow occurrence in the Andes region since 1885». Australian Meterology Magazine, $\mathrm{n}^{\circ}$ 50, 164-168.

RIBBE, L. y GAESE, H. (2002): «Water management issues of the Aconcagua watershed, Chile». Technology Resource Management and Development: Water Management, $\mathrm{n}^{\circ} 2$, 86-108.

RIVERA, A., CASSASA, G., ACUÑA, C. y LANGE, H. (2000): «Variaciones recientes de glaciares en Chile». Revista Investigaciones Geográficas de Chile, n 34, 29-60. 
RIVERA, A., ACUÑA, C., CASSASA, G. y BOWN, F. (2002): «Use of remotely sensed and field data to estimated the contribution of Chilean glaciers to eustatic sea-level rise». Annals of Glaciology, $\mathrm{n}^{\mathrm{o}}$ 34, 367-372.

RIVERA, A. y CASSASA, G. (1999): «Volume changes on Pio XI glacier, Patagonia: 19751995». Global and Planetary Change, $\mathrm{n}^{\circ} 22,233-244$.

RIVERA, A. y CASSASA, G. (2004): «Ice Elevation, Areal, and Frontal Changes of Glaciers from National Park Torres del Paine, Southern Patagonia Icefield». Arctic, Antarctic, and Alpine Research, vol. 36, nº 4, 379-389.

ROSEGRANT, M., RINGLER, C., MCKINNEY, D., CAI, X., KELLER, A. y DONOSO, G. (2000): «Integrated economic-hydrologic water modeling at the basin scale: The Maipo river basin». Agricultural Economic, n $24,33-46$.

TAKAHASHI, K., MONTECINOS, A., GOUBANOVA, K. y DEWITTE, B. (2011): «ENSO regimes: reinterpreting the canonical and Modoki El Niño». Geophysical Research Letters, vol. 38, L10704, doi: 10.1029/2011GL047364

TASCHETTO, A. y ENGLAND, M. (2009): «El Niño Modoki impacts on Australian rainfall». Journal of Climate, vol. 22, $\mathrm{n}^{\circ} 11,3167-3174$.

TRENBERTH, K. (1997): «The definition of El Niño». Bulletin of American Meteorology Society, $\mathrm{n}^{\circ} 78,2771-2777$.

WAYLEN, P. y CAVIEDES, C. (1990): «Annual and seasonal fluctuations of precipitation and streamflow in the Aconcagua river basin, Chile». Journal of Hydrology, $\mathrm{n}^{\circ} 120$, 79-102. 